Pesq. Vet. Bras. 35(Supl.1):1-4, dezembro 2015 DOI: $10.1590 / \mathrm{S} 0100-736 \mathrm{X} 2015001300001$

\title{
Comparação entre duas metodologias para a realização do teste de redução do tetrazólio nitroazul (NBT) em caprinos ${ }^{1}$
}

\author{
Joandes H. Fonteque ${ }^{2 *}$, Mere E. Saito ${ }^{2}$, Priscila A. Rizelo ${ }^{3}$, William T. Teixeira ${ }^{4}$ \\ e Aguemi Kohayagawa ${ }^{5}$
}

\begin{abstract}
Fonteque J.H., Saito M.E., Rizelo P.A., Teixeira W.T. \& Kohayagawa A. 2015. [Comparison among two methodologys at realization nitroblue tetrazolium reduction test (NBT) in caprines.] Comparação entre duas metodologias para a realização do teste de redução do tetrazólio nitroazul (NBT) em caprinos. Pesquisa Veterinária Brasileira 35(Supl.1):1-4. Departamento de Medicina Veterinária, Hospital de Clínicas Veterinárias, Centro de Ciências Agroveterinárias, Universidade do Estado de Santa Catarina, Lages, SC 88520-000, Brazil. E-mail: fonteque@hotmail.com

The nitroblue tetrazolium reduction test (NBT) is one of the most used methods to evaluate the oxidative metabolism of neutrophils. However, the cost for each sample and dye's life-time are disadvantages of the method. This paper aim was to compare two NBT test methodologies in order to maximize its use and decrease the cost for each sample. It was made using 10 adult and healthy goats and blood samples were taken through venipuncion in jugular vein, using $10 \mathrm{~mL}$ tubes, and then a $500 \mu \mathrm{L}$ sample was taken in eppendoff tubes with $2 \mu \mathrm{L}$ of heparin. Using this sample were made three methodologies for the NBT test: A) Park \& Good (1970) original technique; B) dye reduction in 50\% of the volume; C) 50\% reduction and dye storage at $-20^{\circ} \mathrm{C}$ for 180 days. It was made and dyed the blood smear (May Grünwald-Giemsa) from the samples, using the proposed methodologies and then made the counting of 100 neutrophils in optic microscopy, to determinate the percentage of NBT reactive neutrophils. The statistic analysis by the method of Repeated Measuring Analysis (ANOVA) evinced significant differences $(\mathrm{P}<0,05)$ between the two methods and the original $(A=14.4 \pm 4.6 ; B=1.9 \pm 1.4 ; C=1.1 \pm 0.9)$, however it showed high correlation by the Spearman test between the methods ( $r s=0,82(\mathrm{AxB}), \mathrm{rs}=0,75(\mathrm{BxC})$ e $\mathrm{rs}=0.93(\mathrm{AxC})$ ). It was concluded that the original Park \& Good (1970) method and two methodologies for dye reduction in 50\% and it's reduction associated with NBT dye freezing for 180 days can be used in caprines, but the values should be compared with their respective methodologies.
\end{abstract}

INDEX TERMS: Nitroblue tetrazolium reduction test, oxidative metabolism, neutrophil, goats.

RESUMO.- 0 teste de redução do tetrazólio nitroazul (NBT) é um dos métodos mais utilizados para a avaliação do metabolismo oxidativo dos neutrófilos. Porém, o custo por

\footnotetext{
${ }^{1}$ Recebido em 28 de fevereiro de 2015.

Aceito para publicação em 29 de novembro de 2015.

${ }^{2}$ Hospital de Clínica Veterinária (HCV), Departamento de Medicina Veterinária (DMV), Centro de Ciências Agroveterinárias (CAV), Universidade do Estado de Santa Catarina (UDESC), Av. Luiz de Camões 2090, Bairro Conta Dinheiro, Lages, SC 88520-000, Brasil. *Autor para correspondência: fonteque@hotmail.com

3 Bolsista de Iniciação Científica (PROBIC), CAV-UDESC, Lages, SC 88520-000.

${ }^{4}$ Acadêmico de Medicina Veterinária, Departamento de Medicina Veterinária (DMV), CAV-UDESC, Lages, SC 88520-000.

${ }^{5}$ Departamento de Clínica Médica, Faculdade de Medicina Veterinária e Zootecnia (FMVZ), Universidade Estadual Paulista (Unesp), Distrito de Rubião Júnior s/n, Cx. Postal 560, Botucatu, SP 18618-000, Brasil.
}

amostra e o tempo de utilização do corante são desvantagens do método. 0 objetivo deste trabalho foi comparar duas metodologias para realização do teste do NBT a fim de maximizar a sua utilização e diminuir o custo por amostra processada. Foram utilizadas 10 cabras, adultas clinicamente sadias e colhidas amostras de sangue por meio de venipunção jugular em tubos de $10 \mathrm{~mL}$ sendo retirada uma alíquota de $500 \mu \mathrm{L}$ em tubo "eppendorff" contendo $2 \mu \mathrm{L}$ de heparina. Através desta alíquota foram realizadas três metodologias para o teste do NBT: A) técnica original Park \& Good (1970); B) redução de 50\% do volume do corante; C) redução de $50 \%$ e armazenamento do corante a $-20^{\circ} \mathrm{C}$ por 180 dias. Foram confeccionados e corados esfregaços sanguíneos (May Grünwald-Giemsa) das amostras com as metodologias propostas e realizada contagem de 100 neu- 
trófilos em microscopia óptica para determinar o percentual de neutrófilos reativos ao NBT. A análise estatística pelo método de Análise de Medidas Repetida (ANOVA) demonstrou diferenças significativas $(\mathrm{P}<0,05)$ entre os dois métodos e o original $(A=14,4 \pm 4,6 ; B=1,9 \pm 1,4 ; C=1,1 \pm 0,9)$, porém apresentou alta correlação pelo teste de Spearman entre os métodos $\left(r_{s}=0,82(A x B), r_{s}=0,75(B x C)\right.$ e $r_{s}=0,93$ $(A x C))$. Conclui-se que o teste de Redução do Tetrazólio Nitroazul (NBT) segundo a metodologia descrita por Park \& Good (1970) e a redução do corante em 50\% do seu volume e a sua redução associada ao congelamento por 180 dias a $-20^{\circ} \mathrm{C}$ podem ser realizadas em caprinos, porém os valores devem ser comparados com as respectivas metodologias utilizadas.

TERMOS DE INDEXAÇÃO: Teste de redução do tetrazólio nitroazul, metabolismo oxidativo, neutrófilos, caprinos.

\section{INTRODUÇÃO}

Os neutrófilos constituem a primeira linha de defesa do organismo contra a invasão de microorganismos e a sua principal função é fagocitar e destruir bactérias, fungos, leveduras, algas, parasitos e vírus (Park 1971, Roth et al. 1982a, Feldman 2000). Qualquer fator que interfira com a produção ou a supressão das funções dos neutrófilos, rapidamente torna o animal mais susceptível às doenças infecciosas (Roth \& Kaeberle 1982, Feldman 2000). Os mecanismos antibacterianos dos neutrófilos são classificados em dependentes e independentes de oxigênio. 0 mecanismo dependente de oxigênio é iniciado pela fagocitose ou por distúrbios na membrana da célula e envolve uma série de reações que produz espécies reativas de oxigênio (ERO) que interagem com outras substâncias ou atuam diretamente contra as bactérias.

Após a fagocitose realizada pelo neutrófilo ocorre a ativação do sistema NADPH-oxidase que catalisa a reação de redução do oxigênio molecular $\left(\mathrm{O}_{2}\right)$ para ânion superóxido $\left(\mathrm{O}_{2}^{-}\right)$determinando um rápido aumento na atividade da oxidase causando uma "explosão respiratória" no consumo de oxigênio ("respiratory burst") (Ciarlini et al. 2004, Kaneko 2008). Existem muitos métodos para a avaliação do metabolismo oxidativo de neutrófilos. Dentre estes métodos, o teste de redução do tetrazólio nitroazul (NBT) é um dos mais utilizados (Ciarlini 1998, Ciarlini et al. 2004). 0 teste do NBT foi primeiramente utilizado por Baehner \& Nathan (1967) para o diagnóstico de doença granulomatosa crônica (CGD) em crianças.

A CGD é uma doença genética que determina deficiência na produção da enzima NADPH-oxidase de grande importância na geração de ânions superóxido $\left(\mathrm{O}_{2}^{-}\right)$. Desta maneira, a CGD é caracterizada pela presença de infecções bacterianas crônicas recorrentes em humanos (Babior 1978).

O NBT é um corante amarelo claro que quando adicionado a uma suspensão de neutrófilos é reduzido pelo ânion superóxido a um precipitado insolúvel de coloração azul enegrecida conhecido como formazam (Nathan 1974). A redução do NBT tem uma estreita correlação com o metabolismo oxidativo e avalia um importante componente da ação bactericida do neutrófilo (Roth et al. 1983), sendo considerado como uma mensuração indireta da atividade dependente de oxigênio dos neutrófilos (Gordon et al. 1975, Jain 1986). A avaliação é realizada por meio da microscopia óptica e a localização do formazam é muito heterogênea, podendo apresentar-se ligado à membrana da célula ou no interior dos fagócitos (Nathan 1974). Na Medicina Veterinária, Poli et al. (1973) foram os primeiros a utilizarem o teste do NBT em doenças bacterianas de cães. Nos últimos anos, vários autores têm estudado o metabolismo oxidativo de neutrófilos por meio do teste do NBT em diversas situações e espécies de animais, Poli \& Manteli (1974), Sara \& Dwaraknath (1977), Lopes (2000), Fonteque (2001), Lopes et al. (2003) e Souza et al. (2006) em caprinos; Ciarlini (1998), Afonso et al. (2002) e Camargo (2009) em ovinos; Borges et al. (2001), Costa et al. (2000), Peixoto et al. (2002), Costa et al. (2004), Reis et al. (2005), Costa (2005), Ciarlini et al. (2005) e Costa et al. (2008) em bovinos; Ciarlini et al. (1997), Laposy et al. (2000) e Silva et al. (2010) em equinos; Trevelin et al. (2009) em avestruzes; Barbosa et al. (2010), Ciarlini et al. (2010) e Bosco et al. (2012) em caninos. O objetivo do presente trabalho foi comparar duas metodologias para a realização do teste do NBT com o teste padrão desenvolvido por Park \& Good (1970), reduzindo em 50\% o volume do corante e a sua redução em $50 \%$ associada ao armazenamento por 180 dias a temperatura de $-20^{\circ} \mathrm{C}$.

\section{MATERIAL E MÉTODOS}

Foram utilizados 10 caprinos, fêmeas, da raça Saanen, com idade entre oito a 12 meses, pesando entre 28 e $35 \mathrm{Kg}$, clinicamente sadias, livres de ectoparasitas e endoparasitas. Amostras de sangue foram colhidas por venipunção jugular em tubos de $10 \mathrm{~mL}$ a vácuo sem anticoagulante e uma alíquota de $500 \mu \mathrm{L}$ foi retirada e acondicionada em tubo "eppendorff" contendo $2 \mu \mathrm{L}$ de heparina. A mesma amostra de sangue foi utilizada para a realização dos três protocolos do exame. 0 teste foi realizado dentro do período máximo de duas horas após a colheita. A avaliação do metabolismo oxidativo de neutrófilos foi determinada por meio do teste de redução do tetrazólio nitroazul (NBT), método microscópico de Park \& Good (1970), utilizando-se kit comercial (NBT Vial, Sigma Diagnostic. St. Louis, USA). A metodologia utilizada por Park \& Good (1970) constitui-se a utilização de $50 \mu \mathrm{L}$ de NBT e $50 \mu \mathrm{L}$ de sangue (A). A primeira metodologia foi alterada para $25 \mu \mathrm{L}$ de NBT e $25 \mu \mathrm{L}$ de sangue (B); e a segunda metodologia alterada para $25 \mu \mathrm{L}$ de NBT congelado a $-20^{\circ} \mathrm{C}$ por 180 dias e $25 \mu \mathrm{L}$ sangue (C).

Para a realização do método utilizando-se o corante congelado, o NBT foi separado previamente em alíquotas com uma pipeta automática de $25 \mu \mathrm{L}$ em tubos do tipo "eppendorf" e congeladas em freezer à temperatura de $-20^{\circ} \mathrm{C}$ por 180 dias até o momento da realização dos testes. Após a homogeneização as amostras foram incubadas em banho-maria a temperatura de $37^{\circ} \mathrm{C}$ por $10 \mathrm{minu}-$ tos e posteriormente por 10 minutos em temperatura ambiente. Em seguida os frascos foram homogeneizados e confeccionados esfregaços sanguíneos em lâminas de microscopia e corados com corante May Grünwald-Giemsa. A contagem de 100 neutrófilos por meio de microscopia óptica em aumento de $1.000 x$ foi realizada a fim de determinar o número percentual de neutrófilos reativos ao NBT para as três metodologias. Considerou-se neutrófilo NBT positivo quando este apresentou qualquer grânulo intracitoplasmático de coloração azul a negro (formazam), independente do número e tamanho. Os dados foram avaliados por meio do teste de Análise de Variância de Medidas Repetida (ANOVA) compa- 
rando-se os três métodos ao nível de $5 \%$ de significância $(\mathrm{P}<0,05)$ e pela Correlação de Spearman segundo Curi (1998).

\section{RESULTADOS E DISCUSSÃO}

A técnica do NBT é rápida, não envolve equipamentos sofisticados e pode ser realizada a campo (Ciarlini 1998, Fonteque 2001). Porém, o teste implica em algumas limitações, como o custo por amostra e após a diluição do corante sua utilização se restringe a um período de no máximo 24 horas.

A proposta apresentada pelo trabalho visa à possibilidade de redução da quantidade de corante utilizado e a diminuição do custo do teste por amostra processada, além de prolongar a utilização do corante congelado por 180 dias.

A análise estatística dos dados (Quadro 1) demonstrou diferenças significativas $(\mathrm{p}<0,05)$ entre as médias das metodologias propostas e as do método de Park \& Good (1970). Desta maneira a redução em 50\% do corante e sua redução e armazenamento por 180 dias congelado provocam alterações nos resultados do teste de redução do tetrazólio nitroazul (NBT) quando comparados ao método tradicional. Analisando-se as médias dos valores (Quadro 1) os resultados demonstraram que tanto a redução quanto o congelamento do corante não podem ser utilizados como meio de diminuição do custo e de maior utilização do corante. Os valores para as metodologias com redução do corante NBT foram semelhantes aos observados por Lopes (2000) de 4,3\% e Fonteque (2001) que variaram de 0 a $4,0 \%$ para caprinos clinicamente sadios. Porém, os valores de NBT utilizando o método de Park \& Good (1970) estiveram acima dos valores encontrados.

Quadro 1. Valores de média e desvios-padrão ( $\mathrm{x} \pm \mathrm{sd})$ do teste de redução do tetrazólio nitroazul (NBT) em caprinos utilizando os métodos de $50 \mu \mathrm{L}$ fresco segundo Park \& Good

(1970) (A), $25 \mu \mathrm{L}$ de corante fresco (B) e $25 \mu \mathrm{L}$ de corante congelado a $-20^{\circ} \mathrm{C}$ por 180 dias (C)

\begin{tabular}{lrr}
\hline Grupos $\quad 50 \mu \mathrm{L}$ fresco $(\mathrm{A})$ & $25 \mu \mathrm{L}$ fresco $(\mathrm{B})$ & $\begin{array}{l}25 \mu \mathrm{L} \text { congelado } \\
\text { por } 180 \text { dias }(\mathrm{C})\end{array}$ \\
\hline
\end{tabular}

NBT (\%) $\quad 14,4 \pm 4,6^{\text {a }} \quad 1,9 \pm 1,4^{\text {b }} \quad 1,1 \pm 0,9^{\text {b }}$

a Para letras iguais não há diferença significativa $(\mathrm{p}<0,05)$ entre os grupos.

$\mathrm{Na}$ análise das correlações foram observadas altas correlações entre as metodologias segundo o método de Spearman para os respectivos testes: $50 \mu \mathrm{L}$ fresco (A) x $25 \mu \mathrm{L}$ fresco (B) $(r=0,82) ; 25 \mu \mathrm{L}$ fresco (B) x $25 \mu \mathrm{L}$ congelado por 180 dias $(\mathrm{C})(\mathrm{r}=0,75)$ e $50 \mu \mathrm{L}(\mathrm{A})$ x $25 \mu \mathrm{L}$ congelado por 180 dias (C) $(r=0,93)$ (Quadro 2). Os dados obtidos demons-

\begin{tabular}{|c|c|c|}
\hline $50 \mu \mathrm{L}$ fresco $(\mathrm{A}) \times 25 \mu \mathrm{L}$ fresco $(\mathrm{B})$ & 0,82 & 0,0015 \\
\hline $25 \mu \mathrm{L}$ fresco (B) x $25 \mu \mathrm{L}$ congelado por 180 dias (C) & 0,75 & 0,0108 \\
\hline $50 \mu \mathrm{L}(\mathrm{A}) \times 25 \mu \mathrm{L}$ congelado por 180 dias $(\mathrm{C})$ & 0,93 & 0,0000 \\
\hline
\end{tabular}

* Se $\mathrm{p}<0,05$ existe correlação significativa. tram que a alteração na metodologia do teste do NBT pode ser realizada, porém, os valores percentuais relativo aos neutrófilos reativos ao NBT não podem ser comparados com aqueles descritos na literatura que utilizaram o método de Park \& Good (1970). Ciarlini et al (2005) utilizaram a técnica do NBT com redução para $25 \mu \mathrm{l}$ em bovinos vacinados contra a brucelose.

Os valores médios das porcentagens de neutrófilos redutores de NBT antes da vacinação foram semelhantes aos observados por Couto et al. (2001), porém inferiores aos obtidos por Sara e Dwaraknath (1977) e por Costa \& Kohayagawa (2000) para bovinos sadios.

0 estudo demonstrou que a variação do número de neutrófilos reativos ao NBT no momento pré-vacinal esteve entre 0 e $2 \%$, enquanto que os valores descritos por Sara e Dwaraknath (1977) variaram de 2 a 5\%. Os valores de Costa \& Kohayagawa (2000) tiveram média de $6 \%$ para a prova não estimulada e $12 \%$ para a prova estimulada, enquanto no trabalho de Ciarlini et al. (2005) as médias de neutrófilos redutores de NBT para o momento (M0) foram de $0,3 \%$ para a prova não estimulada e $0,8 \%$ para a prova estimulada. Segundo o autor tal diferença deve-se provavelmente ao fato de o tempo de incubação e de os critérios de leitura das reações de redução do NBT utilizados no presente trabalho não serem os mesmos adotados por Costa \& Kohayagawa (2000) e Sara \& Dwaraknath (1977). Porém, não leva em consideração que são duas metodologias distintas, pela redução do volume do corante, podendo ser também incluída como uma das causas da diferença. Trevelin et al. (2009) também utilizaram a técnica com redução para $25 \mu \mathrm{L}$ em avestruzes, porém não puderam comparar com outra literatura pela ausência de trabalhos científicos.

\section{CONCLUSÃO}

Conclui-se que o teste de Redução do Tetrazólio Nitroazul (NBT) segundo a metodologia descrita por Park \& Good (1970) e a redução do corante em $50 \%$ do seu volume e a sua redução associada ao congelamento por 180 dias a $-20^{\circ} \mathrm{C}$ podem ser realizadas em caprinos, porém os valores devem ser comparados com as respectivas metodologias utilizadas.

\section{REFERÊNCIAS}

Afonso J.A.B., Ciarlini P.C., Kuchembuck M.R.G., Kohayagawa A., Feltrin L.H.P.Z., Ciarlini L.D.R.P., Laposy C.B., Mendonça C.L. \& Takahira R.K. 2002. Metabolismo oxidativo de neutrófilos em ovinos tratados com monensina sódica e experimentalmente submetidos à acidose ruminal. Pesq. Vet. Bras. 22(4):129-134.

Babior B.M. 1978. Oxygen-dependent microbial killing by phogocytes. N. Engl. J. Med. 298(13):721-725.

Baehner R.L. \& Nathan D.G. 1967. Leukocyte oxidase: defective activity in chronic granulomatous disease. Science 155:835-836.

Barbosa T.S., Mori C.K. \& Ciarlini P.C. 2010. Efeito inibidor do soro urêmico sobre o metabolismo oxidativo dos neutrófilos de cães. Arq. Bras. Med. Vet. Zootec. 62(6):1352-1358.

Borges A.S., Melchert A., Conceição M., Kohayagawa A., Lopes R.S., Takahira R.K., Kuchembuck M.R.G., Torres L.F. \& Benine L.E. 2001. Metabolismo oxidativo de neutrófilos em bovinos (Bos taurus) durante a premunição contra Anaplasma sp. e Babesia sp. Vet. Notícias 7(1):75-79.

Bosco A.M., Couto R., Pereira P.P., Almeida B.F.M. \& Ciarlini P.C. 2012. Valor 
do teste de redução do tetrazólio nitroazul (NBT) no diagnóstico de processos inflamatórios caninos. Ars Vet. 28(3):161-168.

Camargo E.V. 2009. Atividade de neutrófilos e estresse oxidativo em cordeiros infectados experimentalmente com Haemonchus contortus e suplementados com selênio e vitamina E. Dissertação de Mestrado, Universidade Federal de Santa Maria, Santa Maria. 47p.

Ciarlini P.C., Barros P.C., Lopes C.M.Q., Kohayagawa A., Krause A. \& Laposy C.B. 1997. Leucograma, fibrinogênio plasmático e capacidade bactericida dos neutrófilos de éguas susceptíveis e resistentes à endometrites. Revta Bras. Reprod. Anim. 21:123-126.

Ciarlini P.C. 1998. Leucograma e metabolismo oxidativo dos neutrófilos em ovelhas (Ovis aries Linnaeus, 1758) naturalmente infectadas por nematódeos gastrointestinais ao final da gestação, durante a lactação e após o desmame: Influência de fatores raciais e hormonais. Tese de Doutorado, Faculdade de Medicina Veterinária e Zootecnia, Universidade Estadual Paulista, São Paulo, SP. 204p.

Ciarlini P.C., Patrício R.F., Couto R. \& Bonello F.L. 2004. Efeito da vacina polivalente sobre o leucograma e o metabolismo oxidativo dos neutrófilos em cães. Arqs Inst. Biológico, São Paulo, 71(3):323-327.

Ciarlini P.C., Antonio D.B.A., Barbiere F., Bonello F.L. \& Feitosa F.L.F. 2005. Efeito da vacina contra brucelose bovina sobre a capacidade neutrofílica de redução do NBT. Ars Vet. 21(2):251-256.

Ciarlini P.C., Valadares T.C., Ikeda-Garcia F.A., Marcondes M. \& Lima V.M.F. 2010. Leucograma e metabolismo oxidativo dos neutrófilos de cães com leishmaniose visceral antes e após o tratamento com antimoniato de meglumina e alopurinol. Ciênc. Anim. Bras. 11(2):369-375.

Costa J.N. 2000. Leucograma, metabolismo oxidativo de neutrófilos, proteinograma e imunoglobulinas de bovinos da raça holandesa (Bos taurus). Influência do desenvolvimento etário e da suplementação com vitamina E (acetato de DL- $\alpha$-tocoferol). Tese de Doutorado, Faculdade de Medicina Veterinária e Zootecnia, Universidade Estadual Paulista, Botucatu, SP. 209p.

Costa M.C. 2005. Transferência de Imunidade passiva e metabolismo oxidativo dos neutrófilos em bezerros das raças nelore e limousin. Dissertação de Mestrado, Universidade Estadual de Londrina, Londrina, PR. 84p.

Costa J.N., Peixoto A.P.C., Kohayagawa A., Ferreira A.F.M.S.C.F., Cassetari M.L. \& Crocci A.J. 2004. Influência do desenvolvimento etário e da suplementação com vitamina E (acetato de DL-alfa-tocoferol) no metabolismo oxidativo de neutrófilos de bovinos da raça holandesa. Braz. J. Vet. Res. Anim. Sci. 41:293-298.

Costa M.C., Flaiban K.K.M.C., Coneglian M.M., Feitosa F.L.F., Balarin M.R.S. \& Lisboa J.A.N. 2008. Transferência de imunidade passiva em bezerros das raças nelore e limousine nos primeiros quarto meses de vida. Pesq. Vet. Bras. 28(9):410-416.

Curi P.R. 1998. Metodologia e Análise da Pesquisa em Ciências Biológicas. Tipomic, Botucatu, SP. 263p.

Feldman B.F. 2000. Schalm's Veterinary Hematology. 5th ed. Williams and Wilkins, Philadelphia. 1344p.

Fonteque J.H. 2001. Avaliação dos níveis séricos de cortisol, estradiol e progesterona, do hemograma e do metabolismo oxidativo de neutrófilos em cabras Saanen (Capra hircus) nos períodos do pré e pós-parto. Dissertação de Mestrado em Medicina Veterinária, Área de Clínica Veterinária, Faculdade de Medicina Veterinária e Zootecnia, Universidade Estadual Paulista, Botucatu, SP. 145p.

Gordon P.A., Stuart J., Lee T.R., Breeze G.R. \& Pugh N.H. 1975. The cytocentrifuge NBT test. J. Clin. Pathol. 28(8):674-679.
Jain N.C. 1986. Schalm's Veterinary Hematology. 4th ed. Lea and Febiger, Philadelphia. 1221p.

Kaneko J.J. 2008. Clinical Biochemistry of Domestic Animals. Academic Press, San Diego. 932p.

Laposy C.B., Ballarin M.S., Lopes R.S., Kohayagawa A. \& Takahira R.K. 2000. Avaliação hematológica e metabolismo oxidativo de neutrófilos de eqüinos atletas. Vet. Notícias 6(2):25-29.

Lopes S.T.A. 2000. Perfil leucocitário, atividade funcional neutrofílica, medula óssea, fibrinogênio e proteínas plasmáticas totais em cabras com mastite induzida experimentalmente por Staphylococcus aureus e suplementadas com vitamina E (acetato de DL- $\alpha$-tocoferol). Tese de Doutorado, Faculdade de Medicina Veterinária e Zootecnia, Universidade Estadual Paulista, São Paulo, SP. 159p.

Lopes S.T.A., Paes P.R.O., Kohayagawa A., Lopes R.S., Bulla C. \& Langrafe L. 2003. Atividade functional neutrofílica em cabras com mastite por Staphylococcus aureus e suplementadas com vitamina E (acetate DL- $\alpha$ tocoferol). Arq. Bras. Med. Vet. Zootec. 55(5):515-521.

Nathan D.G. 1974. NBT reduction by human phagocytes. N. Engl. J. Med. 290(5):280-281.

Park B.H. \& Good R.A. 1970. N.B.T. test stimulated. Lancet 296(7728):616.

Park B.H. 1971. The use and limitations of the nitroblue tetrazolium test as a diagnostic aid. J. Pediatrics 78(2):376-378.

Peixoto A.P.C., Costa J.N., Kohayagawa A., Takahira R.K. \& Saito M.E. 2002. Hemograma e metabolismo oxidativo dos neutrófilos de bovinos da raça Holandesa preta e branca: influência dos fatores etários. Revta Bras. Saúde Prod. Anim. 3(1):16-20.

Poli G. \& Mantelli F. 1974. Il 'test' N.B.T. negli animali domestici: valori normali. Clin. Vet., Milano, 97:241-247.

Poli G., Nicoletti G. \& Favarelli G. 1973. Nitroblue tetrazolium (N.B.T.) test nel cane. Folia Vet. Latina 3(2):215-239.

Reis M.C., Costa J.N., Peixoto A.P.C. \& Ferreira A.F.M.S.C. 2005. Efeito da idade e da suplementação oral com o acetato de dl- $\alpha$-tocoferol sobre os níveis séricos de vitamina $\mathrm{E}$ e sobre o metabolismo oxidativo de neutrófilos em bezerros. Revta Bras. Saúde Prod. Anim. 6(1):8-17.

Roth J.A. \& Kaeberle M.L. 1982. Effect of glucocorticoids on the bovine immune system. J. Am. Vet. Med. Assoc. 180(15):894-901.

Roth J.A., Kaeberle M.L., Appell L.H. \& Nachreiner R.F. 1983. Association of increased estradiol and progesterone blood values with altered bovine polymorphonuclear leukocyte function. Am. J. Vet. Res. 44(2):247-253.

Roth J.A., Kaeberle M.L. \& Hsu W.H. 1982a. Effect of estradiol and progesterone on lymphocyte and neutrophil functions in steers. Infect. Immun. 35(3):997-1002.

Sara I.S. \& Dwaraknath P.K. 1977. A note on nitroblue tetrazolium test in normal and diseases animals. Indian J. Anim. Sci. 47(6):362-364.

Silva A.C.R.A., Valadares T.C., Almeida B.F.M. \& Ciarlini P.C. 2010. Efeito do EDTA sobre o teste de redução do tetrazólio nitroazul (NBT) para avaliação do metabolismo oxidativo dos neutrófilos de equinos. Ars Vet. 26(3):124-127.

Souza C., Lopes S.T.A., Batina P.N., Cecim M., Cunha C.M., Conrado A.C. \& Beck A. 2006. Estresse parasitário em cabras Saanen: Avaliação hematológica e da atividade oxidativa dos neutrófilos. Vet. Notícias 12(2):17-23.

Trevelin S.C., Barbosa T.S., Silva V.M.S. \& Ciarlini P.C. 2009. Metabolismo oxidativo de heterófilos de avestruzes jovens. Ciência Rural 39(7):20992104. 